Article

\title{
Anti-Platelet Aggregation and Vasorelaxing Effects of the Constituents of the Rhizomes of Zingiber officinale
}

\author{
Yu-Ren Liao ${ }^{1}$, Yann-Lii Leu ${ }^{2}$, Yu-Yi Chan ${ }^{3}$, Ping-Chung Kuo ${ }^{4}$ and Tian-Shung Wu ${ }^{1,5,6, *}$ \\ 1 Department of Chemistry, National Cheng Kung University, Tainan 701, Taiwan; \\ E-Mail: 13892101@mail.ncku.edu.tw \\ 2 Graduate Institute of Natural Products, Chang Gung University, Taoyuan 333, Taiwan; \\ E-Mail: ylleu@mail.cgu.edu.tw \\ 3 Department of Biotechnology, Southern Taiwan University, Tainan 710, Taiwan; \\ E-Mail: yuyichan@mail.stut.edu.tw \\ 4 Department of Biotechnology, National Formosa University, Yunlin 632, Taiwan; \\ E-Mail: pcckuoo@sunws.nfu.edu.tw \\ 5 Department of Pharmacy, China Medical University, Taichung 404, Taiwan \\ 6 Chinese Medicinal Research and Development Center, China Medical University and Hospital, \\ Taichung 404, Taiwan
}

* Author to whom correspondence should be addressed; E-Mail: tswu@mail.ncku.edu.tw; Tel.: +886-6-275-7575 (ext. 65333); Fax: +886-6-274-0552.

Received: 10 May 2012; in revised form: 13 July 2012 / Accepted: 21 July 2012 /

Published: 26 July 2012

\begin{abstract}
In the present study, the chemical investigation of the bioactive fractions of the rhizomes of Zingiber officinale has resulted in the identification of twenty-nine compounds including one new compound, $O$-methyldehydrogingerol (1). Some of the isolates were subjected into the evaluation of their antiplatelet aggregation and vasorelaxing bioactivities. Among the tested compounds, [6]-gingerol (13) and [6]-shogaol (17) exhibited potent anti-platelet aggregation bioactivity. In addition, [10]-gingerol (15) inhibited the $\mathrm{Ca}^{2+}$-dependent contractions in high $\mathrm{K}^{+}$medium. According to the results in the present research, the bioactivity of ginger could be related to the anti-platelet aggregation and vasorelaxing mechanism.
\end{abstract}

Keywords: anti-platelet aggregation; vasorelaxing; Zingiberaceae; gingerol; shogaol 


\section{Introduction}

Ginger is the rhizome of Zingiber officinale, a perennial plant in the family Zingiberaceae. It is consumed as delicacy, medicine, or spice. It is often pickled in vinegar or sherry as a snack or just cooked as an ingredient in many dishes. There are a variety of uses suggested for ginger as folk medicines in Chinese history, such as stomachic, antiemetic, anti-diarrhea, expectorant, antiasthmatic, hemostatic and cardiotonic for the treatment of several gastrointestinal and respiratory diseases [1]. The most famous traditional medicinal practice of $Z$. officinale is to promote the blood circulation for removing blood stasis and the mechanism is related to anti-platelet aggregation activity [2]. In the course of our continuing research program aimed to discover novel bioactive constituents from natural sources, screening work for thrombolytic and vasoactive activity was carried out and the ether extract of the rhizome of $Z$. officinale was found to show anti-platelet aggregation activity and produce a vasorelaxing effect. This initiated our interest in studying the bioactive principles in the rhizome of Z. officinale. Numerous chemical investigations of the pungent and bioactive principles of $Z$. officinale have been carried out [3,4]. Among these compounds, [6]-gingerol is the major pungent principle of ginger and the chemopreventive potentials present a promising alternative to other toxic therapeutic agents [5]. In the present study, we report the characterization of twenty-nine compounds and the bioactivity of some of these purified compounds.

\section{Results and Discussion}

\subsection{Characterization of the Isolated Compounds}

Air-dried and chopped ginger was filtered and the filtrate was partitioned between ether and water. The residue was extracted with acetone and then also partitioned between ether and water. The ether fractions from the filtrate and residue were combined and concentrated to give a brown syrup. With the assistance of a combination of conventional chromatographic techniques, the ether extract of the Z. officinale afforded one new compound, $O$-methyldehydrogingerol (1), along with twenty eight known compounds, including 1-dehydro-[6]-dehydrogingerol (2) [6], ar-curcumen-15-al (3) [7], 3-(4-hydroxy-3-methoxyphenyl)propionic acid methyl ester (4) [8], curcumin (5) [9], 1 $\beta$-hydroxybisabola-2,10-dien-4-one (6) [10], [6]-dehydrogingerdione (7) [11], [11]-isodehydrogingerdione (8) [12], [6]-, [8]-, and [10]-dehydroshogaol (9, 10, and 11), [6]-gingerdione (12), [6]-, [8]-gingerol (13 and 14) [13], [10]-gingerol (15), [4]-, [6]-, and [8]-shogaol (16, 17, and 18) [13], vanillin (19) [14], dihydrocurcumin (20) [9], methyl-[6]-gingerol (21) [15], ar-curcumene (22) [16], vanillic acid (23) [17], hexahydrocurcumin (24) [18], [6]-gingediol (25) [19], 2,5-dihydroxybisabola-3,10-diene (26) [20], glyceryl-1-hexadecanoate (27) [21], gingerenone-A (28) [22], and cryptomeridiol (29) [23]. The known compounds were identified by comparison of their physical and spectroscopic data with those reported in the literature. The chemical structure of new compound 1 was determined as 1-(4'-hydroxy3'-methoxyphenyl)-5-methoxy-1-decen-3-one with the assistance of NMR and mass spectral analyses and it was given the trivial name $O$-methyldehydrogingerol. Among these isolated compounds, 7, 8, 13-15, 17, 18, and 24-26 (Figure 1) were purified in more quantities and subjected to the bioactivity examinations. 
Figure 1. Structures of compound 1 and the purified constituents subjected into the bioactivity examinations $(\mathbf{7}, \mathbf{8}, \mathbf{1 3}-\mathbf{1 5}, \mathbf{1 7}, \mathbf{1 8}, \mathbf{2 4 - 2 6})$.<smiles>CCCCCC(C)CC(=O)/C=C/c1ccc(O)c(OC)c1</smiles>

1<smiles>CCCCCCCCCC(=O)CC(=O)CCc1ccc(O)c(OC)c1</smiles>

8<smiles>CCCCCCCC(O)CC(=O)CCc1ccc(O)c(OC)c1</smiles>

14<smiles>CCCCC/C=C/C(=O)CCc1ccc(O)c(OC)c1</smiles>

17<smiles>COc1cc(CCC(=O)CC(O)CCc2ccc(O)c(OC)c2)ccc1O</smiles>

24<smiles>CC(C)=CCCC(C)C1CC(O)C=CC1O</smiles>

26<smiles>CCCCC(=O)/C=C(O)/C=C/c1ccc(O)c(OC)c1</smiles><smiles>CCCCCC(O)CC(=O)CCc1ccc(O)c(O)c1</smiles>

13<smiles>CCCCCCCCCC(O)CC(=O)CCc1ccc(O)c(OC)c1</smiles>

15<smiles>CCCCCCC/C=C/C(=O)CCc1ccc(O)c(OC)c1</smiles>

18<smiles>CCCCCCCC(O)CC(O)CCc1ccc(O)c(OC)c1</smiles>

25

\subsection{Anti-Platelet Aggregation Evaluation Bioassay}

Platelets are a natural source of growth factors. They circulate in the blood of mammals and are involved in hemostasis, leading to the formation of blood clots. If the number of platelets is too high, blood clots would form which may obstruct blood vessels and result in some events such as stroke, 
myocardial infarction, and pulmonary embolism or the blockage of blood vessels to other parts of the body, such as the extremities of the arms or legs [24]. The traditional medicinal use of ginger is to promote the blood circulation for removing blood stasis and therefore the compounds isolated in more quantities (compounds 7, 8, 13-15, 17, 18 and 24-26) were selected for the anti-platelet aggregation and vasorelaxing effect bioassay to evaluate their potential to be new lead drugs.

The anti-platelet aggregation results are summarized in Table 1. All the tested compounds displayed significant inhibitory effects on the aggregation of washed rabbit platelets stimulated by the agonists, including arachidonic acid (AA), collagen (Col), platelet activating factor (PAF), and thrombin (Thr). At $100 \mu \mathrm{g} / \mathrm{mL}$ concentration, all the tested compounds except 26 caused complete inhibition of aggregation induced by AA $(100 \mu \mathrm{M})$. Compound $26(100 \mu \mathrm{g} / \mathrm{mL})$ also displayed $67.9 \%$ inhibition of the platelet aggregation. Even at lower concentration, compounds 7, 8, 13, 17, and 18; 14 and 15; 25; and 24, still exhibited excellent inhibitory activity (100\%) against AA-induced platelet aggregation at 1.0, 2.0, 5.0, and $20.0 \mu \mathrm{g} / \mathrm{mL}$, respectively. Compound $\mathbf{1 3}$ displayed the most significant inhibition of platelet aggregation induced by AA with inhibitory percentages of $83.7,66.3,16.7 \%$ at $0.5,0.2$, and $0.1 \mu \mathrm{g} / \mathrm{mL}$, respectively.

Similarly, compounds $\mathbf{7}, \mathbf{8}, \mathbf{1 3}, \mathbf{1 4}, \mathbf{1 5}, \mathbf{1 7}$, and 18 at $100 \mu \mathrm{g} / \mathrm{mL}$ showed the complete inhibition of aggregation induced by $\mathrm{Col}(10 \mu \mathrm{g} / \mathrm{mL})$. However, the inhibitory activities of the tested compounds were not as effective as those induced by AA. At $5.0 \mu \mathrm{g} / \mathrm{mL}$ only compound $\mathbf{1 7}$ displayed excellent inhibition (100\%) against Col-induced platelet aggregation. Compounds $\mathbf{1 3}$ and $\mathbf{1 7}$ exhibited the most significant inhibition of platelet aggregation induced by Col with inhibitory percentages of 15.6 and $28.1 \%$ at $1.0 \mu \mathrm{g} / \mathrm{mL}$, respectively.

In contrast, the tested compounds did not show significant inhibition of aggregation induced by PAF $(2 \mathrm{nM})$ and Thr $(0.1 \mu \mathrm{g} / \mathrm{mL})$. Only compound 17 at $100 \mu \mathrm{g} / \mathrm{mL}$ displayed complete inhibition of aggregation induced by PAF. At $20.0 \mu \mathrm{g} / \mathrm{mL}$ compound 17 only inhibited the platelet aggregation induced by PAF with the percentage of $15.7 \%$. All the tested compounds at $100 \mu \mathrm{g} / \mathrm{mL}$ exhibited the inhibitory percentages lower than $29.5 \%$ against the platelet aggregation induced by Thr.

\subsection{Vasorelaxing Effect Evaluation Bioassay}

The results of inhibition of $\mathrm{KCl}$ - and norepinephrine (NE)-induced contraction of rat aorta were summarized in Table 2. All of the test compounds except 7 at high concentration showed significant inhibition of rat aorta tonic construction induced by $\mathrm{Ca}^{2+}(1.9 \mathrm{mM})$ in high $\mathrm{K}^{+}(80 \mathrm{mM})$ medium. It has been reported that high $\mathrm{K}^{+}$induced contraction in vascular smooth muscle is mediated by an increase in $\mathrm{Ca}^{2+}$ influx through voltage-dependent $\mathrm{Ca}^{2+}$ channels [25]. Among the tested compounds, [10]-gingerol (15) depressed markedly the contractions induced, but not active against the phasic and tonic contractions caused by norepinephrine $(3 \mu \mathrm{M})$. Since 15 inhibited the $\mathrm{Ca}^{2+}$-dependent contractions in high $\mathrm{K}^{+}$medium, it may be a blocker of voltage-dependent $\mathrm{Ca}^{2+}$ channels. 
Table 1. Effects of compounds $7, \mathbf{8}, \mathbf{1 3}-\mathbf{1 5}, \mathbf{1 7}, \mathbf{1 8}, \mathbf{2 4 - 2 6}$ on the aggregation of washed rabbit platelets induced by arachidonic acid (AA), collagen (Col), PAF and thrombin (Thr).

\begin{tabular}{|c|c|c|c|c|c|c|c|c|c|c|c|c|}
\hline \multicolumn{13}{|c|}{ Aggregation (\%) } \\
\hline Inducer & Control & Conc. $(\mu \mathrm{g} / \mathrm{mL})$ & 7 & 8 & 13 & 14 & 15 & 17 & 18 & 24 & 25 & 26 \\
\hline \multirow{10}{*}{$\begin{array}{c}\text { AA } \\
(100 \mu \mathrm{M})\end{array}$} & $85.9 \pm 0.6$ & 0.1 & - & - & $83.3 \pm 2.0$ & & & & - & - & - & \\
\hline & & 0.2 & $85.6 \pm 0.2$ & - & $33.7 \pm 19.1^{\dagger}$ & & & $80.6 \pm 3.6$ & $80.9 \pm 1.7^{\dagger}$ & - & - & \\
\hline & & 0.5 & $17.6 \pm 12.5$ & $82.9 \pm 1.4$ & $16.3 \pm 10.1^{\ddagger}$ & $82.1 \pm 7.3$ & $79.8 \pm 3.8^{\dagger}$ & $16.6 \pm 14.4^{*}$ & $1.4 \pm 1.2^{*}$ & - & $85.6 \pm 1.7$ & \\
\hline & & 1.0 & $0.0 \pm 0.0^{\ddagger}$ & $0.0 \pm 0.0^{*}$ & $0.0 \pm 0.0^{*}$ & $18.1 \pm 3.8^{*}$ & $2.7 \pm 2.3^{\ddagger}$ & $0.0 \pm 0.0^{\ddagger}$ & $0.0 \pm 0.0^{*}$ & - & $44.4 \pm 21.1 *$ & \\
\hline & & 2.0 & - & - & - & $0.0 \pm 0.0^{\ddagger}$ & $0.0 \pm 0.0^{*}$ & & - & $86.1 \pm 0.7$ & $34.9 \pm 18.3^{*}$ & \\
\hline & & 5.0 & - & - & - & & & & - & $41.5 \pm 20.8 *$ & $0.0 \pm 0.0^{*}$ & \\
\hline & & 10.0 & - & - & - & & & & - & $27.9 \pm 16.4^{\ddagger}$ & - & \\
\hline & & 20.0 & - & - & - & & & & - & $0.0 \pm 0.0^{\ddagger}$ & - & \\
\hline & & 50.0 & - & - & - & & & & - & - & - & $84.8 \pm 0.5$ \\
\hline & & 100.0 & $0.0 \pm 0.0^{*}$ & $0.0 \pm 0.0^{\ddagger}$ & $0.0 \pm 0.0^{ \pm}$ & $0.0 \pm 0.0^{\ddagger}$ & $0.0 \pm 0.0^{\ddagger}$ & $0.0 \pm 0.0^{\ddagger}$ & $0.0 \pm 0.0^{ \pm}$ & $0.0 \pm 0.0^{\ddagger}$ & $0.0 \pm 0.0^{*}$ & $32.1 \pm 16.7^{\dagger}$ \\
\hline \multirow{8}{*}{$\begin{array}{c}\text { Col } \\
(10 \mu \mathrm{g} / \mathrm{mL})\end{array}$} & $87.1 \pm 1.0$ & 0.5 & & & & & & $83.3 \pm 0.7 *$ & - & - & & \\
\hline & & 1.0 & - & - & $84.4 \pm 2.9$ & & & $71.9 \pm 4.7^{\ddagger}$ & - & - & & \\
\hline & & 2.0 & $78.5 \pm 2.7$ & - & $69.6 \pm 9.9^{\dagger}$ & & $84.2 \pm 3.7$ & $30.6 \pm 10.8^{*}$ & $82.5 \pm 4.0$ & & & \\
\hline & & 5.0 & $37.4 \pm 15.6^{*}$ & $86.3 \pm 1.3$ & $40.6 \pm 17.9^{\ddagger}$ & $76.8 \pm 4.8^{\dagger}$ & $77.7 \pm 1.9^{*}$ & $0.0 \pm 0.0^{\ddagger}$ & $25.4 \pm 14.0^{*}$ & & & \\
\hline & & 10.0 & $4.6 \pm 4.0^{\ddagger}$ & $54.8 \pm 18.0 *$ & $12.6 \pm 10.3^{\ddagger}$ & $29.0 \pm 16.9^{*}$ & $20.6 \pm 10.6^{\ddagger}$ & & $0.0 \pm 0.0^{\ddagger}$ & & $80.1 \pm 5.1$ & \\
\hline & & 20.0 & $0.0 \pm 0.0^{*}$ & $4.6 \pm 4.0^{*}$ & $0.0 \pm 0.0^{\ddagger}$ & $0.0 \pm 0.0^{\ddagger}$ & $0.0 \pm 0.0^{\ddagger}$ & & & $77.6 \pm 4.0^{\dagger}$ & $54.7 \pm 15.6 *$ & \\
\hline & & 50.0 & - & $0.0 \pm 0.0^{\ddagger}$ & - & & & & & $33.0 \pm 17.2^{*}$ & $47.2 \pm 13.7^{\ddagger}$ & \\
\hline & & 100.0 & $0.0 \pm 0.0^{*}$ & $0.0 \pm 0.0^{*}$ & $0.0 \pm 0.0^{*}$ & $0.0 \pm 0.0^{*}$ & $0.0 \pm 0.0^{*}$ & $0.0 \pm 0.0^{\ddagger}$ & $0.0 \pm 0.0^{*}$ & $3.8 \pm 3.3^{\ddagger}$ & $15.5 \pm 8.9^{\ddagger}$ & $89.5 \pm 1.8$ \\
\hline \multirow{3}{*}{$\begin{array}{c}\text { PAF } \\
(2 \mathrm{nM})\end{array}$} & $88.6 \pm 1.8$ & 20.0 & & & & & & $84.3 \pm 3.6$ & & & & \\
\hline & & 50.0 & $83.9 \pm 1.0$ & $83.0 \pm 1.0 *$ & & $83.2 \pm 1.4$ & $83.4 \pm 1.6$ & $59.9 \pm 8.5^{\dagger}$ & $84.9 \pm 4.0$ & & & \\
\hline & & 100.0 & $55.8 \pm 4.7^{*}$ & $55.3 \pm 11.6^{\dagger}$ & & $24.4 \pm 8.6^{*}$ & $10.5 \pm 8.6^{*}$ & $0.0 \pm 0.0^{\ddagger}$ & $55.8 \pm 9.2^{\dagger}$ & $84.5 \pm 2.4$ & $84.9 \pm 1.5$ & $85.6 \pm 1.3$ \\
\hline $\begin{array}{c}\text { Thr } \\
(0.1 \mu \mathrm{g} / \mathrm{mL})\end{array}$ & $92.3 \pm 1.0$ & 100.0 & $87.4 \pm 1.1$ & $84.5 \pm 0.6^{ \pm}$ & & $85.0 \pm 0.5^{*}$ & $84.3 \pm 2.5 *$ & $70.5 \pm 2.7^{*}$ & $80.2 \pm 0.9^{*}$ & $85.1 \pm 4.7$ & $88.5 \pm 1.1 *$ & $88.2 \pm 3.6$ \\
\hline
\end{tabular}

Platelets were preincubated with compounds or DMSO $\left(0.5 \%\right.$, control) at $37{ }^{\circ} \mathrm{C}$ for $3 \mathrm{~min}$; the inducer was then added. Values were means \pm s.e.m. $(\mathrm{n}=3-7) .{ }^{*} p<0.05,{ }^{\dagger} p<0.01$,

${ }^{\ddagger} p<0.001$ as compared with the control. 
Table 2. Inhibition of $\mathrm{KCl}$, norepinephrine-induced contraction of rat aorta by compounds $7,8,13-15,17,18,24-26$.

\begin{tabular}{|c|c|c|c|c|}
\hline \multirow[t]{2}{*}{ Compound } & \multirow[t]{2}{*}{$\begin{array}{l}\text { Conc. } \\
(\mu \mathrm{M})\end{array}$} & \multicolumn{2}{|c|}{$\begin{array}{c}\mathrm{NE}(3 \mu \mathrm{M}) \\
\text { contraction }(\%)\end{array}$} & \multirow{2}{*}{$\begin{array}{c}\mathrm{K}^{+}(80 \mathrm{mM})+\mathrm{Ca}^{2+}(1.9 \mathrm{mM}) \\
\text { contraction }(\%)\end{array}$} \\
\hline & & Phasic & Tonic & \\
\hline Control & & $100.0 \pm 5.1$ & $100.0 \pm 4.1$ & $100.0 \pm 4.9$ \\
\hline 7 & 362 & $86.6 \pm 1.6$ & $84.7 \pm 1.5$ & $75.4 \pm 3.2$ \\
\hline \multirow[t]{2}{*}{8} & 287.4 & $100.0 \pm 0.0$ & $85.3 \pm 2.8$ & $46.9 \pm 4.2$ \\
\hline & 86.2 & - & - & $72.3 \pm 3.7$ \\
\hline \multirow[t]{3}{*}{13} & 357.1 & $49.2 \pm 2.3$ & $40.3 \pm 3.8$ & $13.4 \pm 1.1$ \\
\hline & 107.1 & $88.7 \pm 1.9$ & $93.3 \pm 0.0$ & $26.4 \pm 2.4$ \\
\hline & 35.7 & - & - & $96.7 \pm 1.9$ \\
\hline \multirow[t]{3}{*}{14} & 310.6 & $63.3 \pm 3.1$ & $48.1 \pm 3.9$ & $12.5 \pm 0.0$ \\
\hline & 93.2 & $89.9 \pm 9.2$ & $73.2 \pm 2.8$ & $20.9 \pm 2.1$ \\
\hline & 31.1 & - & - & $83.2 \pm 4.9$ \\
\hline \multirow[t]{3}{*}{15} & 285.7 & $104.2 \pm 1.9$ & $65.1 \pm 7.2$ & $23.8 \pm 3.1$ \\
\hline & 85.7 & - & - & $48.1 \pm 0.9$ \\
\hline & 28.6 & - & - & $68.6 \pm 4.9$ \\
\hline \multirow[t]{3}{*}{17} & 362.3 & $60.0 \pm 14.1$ & $36.7 \pm 2.4$ & $11.0 \pm 0.4$ \\
\hline & 108.7 & $90.3 \pm 0.9$ & $89.9 \pm 1.7$ & $29.7 \pm 3.8$ \\
\hline & 36.2 & - & - & $83.0 \pm 1.1$ \\
\hline \multirow[t]{3}{*}{18} & 328.9 & $109.7 \pm 3.7$ & $78.6 \pm 2.8$ & $24.3 \pm 4.3$ \\
\hline & 98.7 & - & - & $58.0 \pm 1.9$ \\
\hline & 32.9 & - & - & $93.2 \pm 1.9$ \\
\hline \multirow[t]{2}{*}{24} & 267.4 & $76.7 \pm 10.0$ & $72.2 \pm 3.2$ & $50.9 \pm 7.8$ \\
\hline & 80.2 & - & - & $95.0 \pm 4.9$ \\
\hline \multirow[t]{2}{*}{25} & 308.6 & $78.3 \pm 2.3$ & $52.7 \pm 2.9$ & $12.0 \pm 1.0$ \\
\hline & 92.6 & $83.9 \pm 1.8$ & $83.5 \pm 4.1$ & $71.7 \pm 1.8$ \\
\hline \multirow[t]{2}{*}{26} & 446.4 & $72.5 \pm 1.8$ & $53.0 \pm 1.3$ & $45.1 \pm 5.1$ \\
\hline & 133.9 & $80.0 \pm 0.0$ & $75.3 \pm 3.8$ & $88.6 \pm 7.8$ \\
\hline
\end{tabular}

Rat aorta rings were preincubated with compounds or DMSO $\left(1 \%\right.$, control) at $37^{\circ} \mathrm{C}$ for $15 \mathrm{~min}$, the inducer was then added. Values were means \pm s.e.m. "“-": no test.

\subsection{Structure-Bioactivity Relationships}

From the above bioassay data, [6]-gingerol (13) and [6]-shogaol (17) exhibited more potent anti-platelet aggregation bioactivity. We could conclude that the compounds which possessed a carbonyl groupa at the 6-position of the alkyl chain in phenylalkanoids and phenylalkenoids would be good drug lead candidates. According to the purified constituents and bioactivity in the present research, the function of ginger to promote the blood circulation for removing blood stasis could be related to anti-platelet aggregation and vasorelaxing mechanism. 


\section{Experimental}

\subsection{General Procedures}

All the chemicals were purchased from Merck KGaA (Darmstadt, Germany) unless specifically indicated. Melting points of purified compounds were determined by a Yanagimoto micromelting point measuring apparatus (Tokyo, Japan) without corrections. The UV spectra were obtained on a Hitachi UV-3210 spectrophotometer (Tokyo, Japan). The IR spectra were obtained as KBr discs on a Jasco Report-100 FT-IR spectrometer (Tokyo, Japan). Optical rotations were measured with the Jasco DIP-370 Digital polarimeter (Tokyo, Japan). The mass and high-resolution mass spectra were obtained on a VG-70-250S mass spectrometer equipped with a direct inlet system. ${ }^{1} \mathrm{H}-,{ }^{13} \mathrm{C}$-, and $2 \mathrm{D}-\mathrm{NMR}$ spectra were recorded on the Bruker AC-200, AMX-400 (Bruker Biospin Inc., Ettlingen, Germany) and Varian Unity (Palo Alto, CA, USA) plus $400 \mathrm{MHz}$ spectrometers with tetramethylsilane as the internal standard. Standard pulse sequences and parameters were used for the NMR experiments and all chemical shifts were reported in parts per million (ppm, $\delta$ ). Column chromatography was performed on silica gels (Kieselgel 60, 70-230 mesh and 230-400 mesh, Merck KGaA, Darmstadt, Germany). Thin layer chromatography (TLC) was conducted on precoated Kieselgel $60 \mathrm{~F} 254$ plates (Merck) and the compounds were visualized by UV light or spraying with $10 \%(\mathrm{v} / \mathrm{v}) \mathrm{H}_{2} \mathrm{SO}_{4}$ followed by heating at $110^{\circ} \mathrm{C}$ for $10 \mathrm{~min}$.

\subsection{Isolation}

The chopped rhizomes of $Z$. officinale $(64.4 \mathrm{~kg})$ were filtered to afford the ginger juice and the residue. The filtrate was partitioned between ether $\left(\mathrm{Et}_{2} \mathrm{O}\right)$ and water $\left(\mathrm{H}_{2} \mathrm{O}\right)$, whereas the residue was extracted with acetone five times. The acetone extracts were combined, concentrated, and then also partitioned between $\mathrm{Et}_{2} \mathrm{O}$ and $\mathrm{H}_{2} \mathrm{O}$. The ether solutions from the filtrate and residue were combined and concentrated again to give a brown syrup $(623 \mathrm{~g})$ which was subjected to silica gel column chromatography using a gradient of benzene $\left(\mathrm{C}_{6} \mathrm{H}_{6}\right)$ and acetone $\left(\mathrm{Me}_{2} \mathrm{CO}\right)$ as eluent to yield twelve fractions. Frs 1-4 were mixed together and rechromatographed over silica gel developing with $\mathrm{C}_{6} \mathrm{H}_{6}-\mathrm{Me}_{2} \mathrm{CO}$ (9:1) to give 1 (5 mg), 2 (2 mg), 7 (295 mg), 8 (4 mg), and 12 (7 mg), successively. The combination of Frs 5-8 was repeated chromatographed over silica gel and thin layer chromatography (TLC) to yield 3 (2 mg), 4 (2 mg), 6 (3 mg), 9 (7 mg), 10 (3 mg), 11 (2 mg), 13 (18.6 mg), 14 (675 mg), $15(96 \mathrm{mg}), \mathbf{1 7}(18 \mathrm{mg}), \mathbf{1 8}(7 \mathrm{mg}), \mathbf{1 9}(2 \mathrm{mg}), \mathbf{2 0}(23 \mathrm{mg})$, and $21(22 \mathrm{mg})$ from the subfractions, respectively. Frs 9-11 were also combined and rechromatographed over silica gel eluting with $\mathrm{CHCl}_{3}-\mathrm{Me}_{2} \mathrm{CO}$ (9:1) to afford 16 (4 mg), 22 (2 mg), 23 (5 mg), 24 (530 mg), 25 (2 mg), 26 $(60 \mathrm{mg}), 27(2 \mathrm{mg})$ and $28(3 \mathrm{mg})$, successively. The last fraction was rechromatographed on RP-18 column eluting with $\mathrm{H}_{2} \mathrm{O}$ and step gradient of methanol to yield $5(2 \mathrm{mg})$ and $29(3 \mathrm{mg})$.

\subsection{Antiplatelet Aggregatory and Vasorelaxing Activity Bioassays}

Assays of the antiplatelet aggregatory and vasorelaxing activities of isolates were done according to the procedures of Teng and coworkers [26,27]. Washed platelets were prepared from blood withdrawn with a siliconized syringe from the marginal vein of New Zealand rabbits. The platelet suspension 
was obtained from EDTA-anticoagulated platelet-rich plasma according to the washing procedure described previously. Platelet number was counted with a cell counter (Hema-laser 2, Sebia, France) and adjusted to $3.0 \times 10^{8}$ platelets $/ \mathrm{mL}$. The platelet pellets were suspended in Tyrode's solution containing $\mathrm{Ca}^{2+}(1 \mathrm{mM})$ and bovine serum albumin $(0.35 \%)$. All glassware was siliconized. Platelet aggregation was measured by the turbidimetric method [27]. The aggregations were measured with a Lumi-aggregometer (Model 1020, Payton, Stouffville, Canada) connected to two dual-channel recorders.

\subsection{Spectral Data}

O-Methyldehydrogingerol (1): colorless syrup; $[\alpha]_{\mathrm{D}}-30.2^{\circ}\left(\mathrm{c} 0.02, \mathrm{CHCl}_{3}\right)$; $\mathrm{UV} \lambda \max 228,290$ (sh), $341 \mathrm{~nm}$; IR $v_{\max } 3450,1651,1589,1515 \mathrm{~cm}^{-1} ;{ }^{1} \mathrm{H}-\mathrm{NMR}\left(\mathrm{CDCl}_{3}, 400 \mathrm{MHz}\right) \delta 0.88(3 \mathrm{H}, \mathrm{t}, J=6.5 \mathrm{~Hz}$, H-10), 1.2-2.4 (8H, m, H-6 to H-9), 2.65 (1H, dd, $J=15.6,5.2 \mathrm{~Hz}, \mathrm{H}-4 \mathrm{a}), 2.95$ (1H, dd, $J=15.6$, $7.6 \mathrm{~Hz}, \mathrm{H}-4 \mathrm{~b}), 3.35$ (3H, s, $\left.\mathrm{OCH}_{3}-5\right), 3.77$ (1H, m, H-5), 3.94 (3H, s, OCH $\left.{ }_{3}-3^{\prime}\right), 5.90$ (1H, br s, OH-4'), $6.63(1 \mathrm{H}, \mathrm{d}, J=16.0 \mathrm{~Hz}, \mathrm{H}-2), 6.93(1 \mathrm{H}, \mathrm{d}, J=8.0 \mathrm{~Hz}, \mathrm{H}-5 '), 7.06\left(1 \mathrm{H}, \mathrm{d}, J=2.0 \mathrm{~Hz}, \mathrm{H}-2^{\prime}\right), 7.11(1 \mathrm{H}$, $\mathrm{dd}, J=8.0,2.0 \mathrm{~Hz}, \mathrm{H}-6$ '), $7.50(1 \mathrm{H}, \mathrm{d}, J=16.0 \mathrm{~Hz}, \mathrm{H}-1) ;{ }^{13} \mathrm{C} \mathrm{NMR}\left(\mathrm{CDCl}_{3}, 100 \mathrm{MHz}\right) \delta 14.1(\mathrm{C}-10)$, 22.6 (C-9), 29.1 (C-7), 29.5(C-8), 32.5 (C-6), 41.7 (C-4), $55.9\left(\mathrm{OCH}_{3}-3\right.$ '), $56.3\left(\mathrm{OCH}_{3}-5\right), 76.8(\mathrm{C}-5)$, 114.4 (C-1'), 119.6 (C-2'), 127.8 (C-5'), 130.2 (C-2), 134.5 (C-6'), 144.8 (C-1), 145.4 (C-4'), 147.7 (C-3'), $199.6(\mathrm{C}=\mathrm{O})$; EIMS m/z (rel. int.) $306\left(\mathrm{M}^{+}, 21\right), 235$ (9), 177 (100), 150 (53), 145 (18), 137 (17), 117 (9), 55 (12); HREIMS m/z 306.1838 [M] (calcd for $\mathrm{C}_{18} \mathrm{H}_{26} \mathrm{O}_{4}, 306.1831$ ).

\section{Conclusion}

In summary, twenty-nine compounds were isolated from the rhizomes the Z. officinale. The pharmacological activities including anti-platelet aggregation and vasorelaxing effects have been documented. However, dose and the form in which they should be used require further standardization. All these are due to the synergistic effects of zingiberene and related types of components bring about the pharmacological impact. In conclusion, ginger has a wide range of medicinal uses and can be used either as a single drug or compound drugs to treat different ailments.

\section{Acknowledgments}

We thank National Science Council, R.O.C. for support of this research. We also thank C. S. Kuoh for the plant collection and identification. This study is supported in part by Taiwan Department of Health Clinical Trial and Research Center of Excellence (DOH100-TD-B-111-004).

\section{References}

1. Tang, W.; Eisenbrand, G. Chinese Drugs of Plant Origin; Springer-Verlag: Berlin, Germany, 1992; pp. 1011-1052.

2. Nurtjahja-Tjendraputra, E.; Ammit, A.J.; Roufogalis, B.D.; Tran, V.H.; Duke, C.C. Effective anti-platelet and COX-1 enzyme inhibitors from pungent constituents of ginger. Thromb. Res. 2003, 111, 259-265.

3. Wu, T.S.; Wu, Y.C.; Wu, P.L.; Chern, C.H.; Leu, Y.L.; Chan, Y.Y. Structure and synthesis of [n]-dehydroshogaols from Zingiber officinale. Phytochemistry 1998, 48, 889-891. 
4. Tao, Q.F.; Xu, Y.; Lam, R.Y.Y.; Schneider, B.; Dou, H.; Leung, P.S.; Shi, S.Y.; Zhou, C.X.; Yang, L.X.; Zhang, R.P.; et al. Diarylheptanoids and a monoterpenoid from the rhizomes of Zingiber officinale: Antioxidant and cytoprotective properties. J. Nat. Prod. 2008, 71, 12-15.

5. Shukla, Y.; Singh, M. Cancer preventive properties of ginger: A brief review. Food Chem. Toxicol. 2007, 45, 683-687.

6. Jolad, S.D.; Lantz, R.C.; Chen, G.J.; Bates, R.B. Commercially processed dry ginger (Zingiber officinale): Composition and effects on LPS-stimulated $\mathrm{PGE}_{2}$ production. Phytochemistry 2005, 6 , 1614-1617.

7. Weyerstahl, P.; Schlicht, V. Synthesis of rac-ar-curcumen-15-al. Liebigs Ann. 1997, 1, 175-178.

8. Hsieh, P.W.; Chang, F.R.; Wu, C.C.; Li, C.M.; Wu, K.Y.; Chen, S.L.; Yen, H.F.; Wu, Y.C. Longicalycinin A, a new cytotoxic cyclic peptide from Dianthus superbus var. longicalycinus (Maxim.) Will. Chem. Pharm. Bull. 2005, 53, 336-339.

9. Uehara, S.I.; Yasuda, I.; Akiyama, K.; Morita, H.; Takeya, K.; Itokawa, H. Diarylheptanoids from the rhizomes of Curcuma xanthorrhiza and Alpinia officinarum. Chem. Pharm. Bull. 1987, 35, 3298-3302.

10. Castro, V.; Tamayo-Castillo, G.; Jakupovic, J. Sesquiterpene lactones and other constituents from Calea prunifolia and C. Peckii. Phytochemistry 1989, 28, 2415-2418.

11. Kiuchi, F.; Iwakami, S.; Shibuya, M.; Hanaoka, F.; Sankawa, U. Inhibition of prostaglandin and leukotriene biosynthesis by gingerols and diarylheptanoids. Chem. Pharm. Bul. 1992, 40, 387-391.

12. Shoji, N.; Iwasa, A.; Takemoto, T.; Ishida, Y.; Ohizumi, Y. Cardiotonic principles of ginger (Zingiber officinale Roscoe). J. Pharm. Sci. 1982, 71, 1174-1179.

13. Hatanaka, M.; Himeda, Y.; Imashiro, R.; Tanaka, Y.; Ueda, I. Allylidenetriphenylphosphorane as a bifunctional reagent: Synthesis of cyclopentenones and $\alpha, \beta$-unsaturated ketones with (3-(alkoxycarbonyl)-2-ethoxy-2-propenylidene)triphenylphosphorane. J. Org. Chem. 1994, 59, 111-115.

14. Wu, T.S.; Tsang, Z.J.; Wu, P.L.; Lin, F.W.; Li, C.Y.; Teng, C.M.; Lee, K.H. New constituents and antiplatelet aggregation and anti-HIV principles of Artemisia capillaris. Bioorg. Med. Chem. 2001, 9, 77-83.

15. Denniff, P.; Macleod, I.; Whiting, D.A. Studies in the biosynthesis of [6]-gingerol, pungent principle of ginger (Zingiber officinale). J. Chem. Soc. Perkin Trans. 1 1980, 2637-2641.

16. Hagiwara, H.; Okabe, T.; Ono, H.; Kamat, V.P.; Hoshi, T.; Suzuki, T.; Ando, M. Total synthesis of bisabolane sesquiterpenoids, $\alpha$-bisabol-1-one, curcumene, curcuphenol and elvirol: Utility of catalytic enamine reaction in cyclohexenone synthesis. J. Chem. Soc. Perkin Trans. 1 2002, 895-899.

17. Wu, P.L.; Su, G.C.; Wu, T.S. Bioactive constituents from the stems of Aristolochia manshuriensis. J. Nat. Prod. 2003, 66, 996-998.

18. Ohtsu, H.; Xiao, Z.; Ishida, J.; Nagai, M.; Wang, H.K.; Itokawa, H.; Su, C.Y.; Shin, C.; Chiang, T.; Chang, E.; et al. Antitumor agents. 217. Curcumin analogues as novel androgen receptor antagonists with potential as anti-prostate cancer agents. J. Med. Chem. 2002, 45, 5037-5041.

19. Kikuzaki, H.; Tsai, S.M.; Nakatani, N. Gingerdiol related compounds from the rhizomes of Zingiber officinale. Phytochemistry 1992, 31, 1783-1785.

20. Ohshiro, M.; Kuroyanagi, M.; Ueno, A. Structures of sesquiterpenes from Curcuma longa. Phytochemistry 1990, 29, 2201-2204. 
21. Rejzek, M.; Vacek, M.; Wimmer, Z. A mild approach to the synthesis of sn-Glycerol 1,2-Di- $\gamma$-linolenate 3-palmitate. Helv. Chim. Acta 2000, 83, 2756-2759.

22. Kikuzaki, H.; Usuguchi, J.; Nakatani, N. Constitutents of Zingiberaceae. I. Diarylheptanoids from the rhizomes of ginger (Zingiber officinale Roscoe). Chem. Pharm. Bull. 1991, 39, 120-125.

23. Wu, S.L.; Li, W.S. Chemical constituents from the roots of Neolitsea hiiranensis. J. Chin. Chem. Soc. 1997, 42, 555-559.

24. Wu, T.S.; Chern, H.J.; Damu, A.G.; Kuo, P.C.; Su, C.R.; Lee, E.J.; Teng, C.M. Flavonoids and andrographolides from Andrographis paniculata and their antiplatelet aggregatory and vasorelaxing effects. J. Asian Nat. Prod. Res. 2008, 10, 17-24.

25. Guh, J.H.; Ko, F.N.; Yu, S.M.; Wu, Y.C.; Teng, C.M. Pharmacological evaluation of N-methylactinodaphnine, a new vascular $\alpha$-adrenoceptor antagonist, isolated from Illigera luzonensis. Eur. J. Pharm. 1995, 279, 33-41.

26. Teng, C.M.; Chen, W.C.; Ko, F.N.; Lee, L.G.; Huang, T.F.; Chen, Y.P.; Hsu, H.Y. Two antiplatelet agents from Magnolia officinalis. Thromb. Res. 1988, 50, 757-765.

27. Born, G.V.R.; Cross, M.J. Effects of inorganic ions and of plasma proteins on the aggregation of blood platelets by adenosine diphosphate. J. Physiol. 1963, 168, 178-195.

Sample Availability: Samples of the Z. officinale are available from the authors.

(C) 2012 by the authors; licensee MDPI, Basel, Switzerland. This article is an open access article distributed under the terms and conditions of the Creative Commons Attribution license (http://creativecommons.org/licenses/by/3.0/). 